\title{
Polaronic distortion and vacancy-induced magnetism in MgO
}

\author{
A. Droghetti, C. D. Pemmaraju, and S. Sanvito \\ School of Physics and CRANN, Trinity College, Dublin 2, Ireland \\ (Received 4 February 2010; published 12 March 2010)
}

\begin{abstract}
The electronic structure of the neutral and singly charged $\mathrm{Mg}$ vacancy in $\mathrm{MgO}$ is investigated using density functional theory. For both defects, semilocal exchange correlation functionals such as the local spin density approximation incorrectly predict a delocalized degenerate ground state. In contrast, functionals that take strong correlation effects into account predict a localized solution, in agreement with spin resonance experiments. Our results, obtained with the HSE hybrid, atomic self-interaction corrected and LSDA $+U$ functionals, provide a number of constraints to the possibility of ferromagnetism in hole doped $\mathrm{MgO}$.
\end{abstract}

DOI: 10.1103/PhysRevB.81.092403

PACS number(s): 75.30.Hx, 71.27.+a, 75.50.Pp

In recent years, the so-called " $d^{0}$ magnets" have attracted great interest within the magnetism community and have been the topic of many theoretical and experimental investigations. Broadly speaking, the term $d^{0}$ magnets refers to a class of materials which, lacking any magnetic ions with open $d$ or $f$ shells, should in principle not be ferromagnetic, but nevertheless exhibit signatures of ferromagnetism often with a Curie temperature exceeding $300 \mathrm{~K}^{1}{ }^{1}$ Defective graphite $^{2}$ and gold nanoparticles capped with organic molecules ${ }^{3}$ are classical examples. However, the most widely studied class of $d^{0}$ magnets is that of undoped or lightelement doped medium to wide-gap oxides. The first experimental study, which boosted research in this field, was on undoped $\mathrm{HfO}_{2}{ }^{4}$ Since then, there have been similar reports of ferromagnetism for numerous other oxides such as $\mathrm{TiO}_{2}{ }^{5}$ $\mathrm{SnO}_{2},{ }^{6} \mathrm{CeO}_{2},{ }^{7} \mathrm{ZnO},{ }^{8} \mathrm{CuO}_{2},{ }^{9} \mathrm{MgO}^{10-12}$ etc. Generally, the ferromagnetism is observed in highly defective samples leading to the expectation that the magnetism must be somehow defect-related. Nanostructures are also often reported ferromagnetic and it has been argued that ferromagnetism can be a general property of nanoparticles. ${ }^{15}$ Nevertheless the precise mechanism behind the reported high temperature ferromagnetism is not well understood and is a subject of open debate.

On the theoretical front, $a b$ initio calculations based on Density Functional Theory (DFT) have been widely employed to study $d^{0}$ magnetism in a wide range of systems. Following the early work of Elfimov et al. on $\mathrm{CaO},{ }^{16}$ and in parallel with many experimental studies, a number of theoretical publications, proposing new $d^{0}$ ferromagnetic materials, have appeared over the past few years. In most of the $d^{0}$ oxides proposed, the formation of magnetic moments and the resulting ferromagnetism is attributed to spin-polarized holes residing on cation $p$ orbitals either at vacancy or impurity sites. ${ }^{16,17}$ Critically however, almost all such predictions are based on DFT calculations performed with local approximations of the exchange correlation potentials, such as the local spin density approximantion (LSDA) and the generalized gradient approximation (GGA). These notoriously suffer from the spurious self-interaction, whose consequence is that of overdelocalizing the charge density. It is then not surprising that most of the calculations return a metallic (often halfmetallic) ground state and usually, extremely large magnetic interaction. It has been shown previously that correcting for self-interaction can lead to the holes states localizing as a consequence of lattice distortions and the magnetic coupling is then reduced drastically or even completely suppressed. ${ }^{18,19}$ This is consistent with the very well established notion that holes bound to acceptor defects form small polarons. ${ }^{20,21}$ More recently, the electronic properties of cation vacancies in many $\mathrm{Zn}$ chalcogenides was investigated in detail by Chan et al. ${ }^{22}$ It was shown that the metallic band structure predicted by LSDA is changed into an insulating one as soon as a hole-state potential operator, ${ }^{23}$ which increases the splitting between occupied and unoccupied anion $p$ states, is applied. Pardo et al. ${ }^{24}$ investigated the effect of on-site $U$ corrections on the $p$-orbital magnetism in $N$ doped alkaline earth monoxides and showed that for realistic values of the $U$ parameter, the ferromagnetic coupling is drastically diminished.

Without entering into the issues connected with percolation, ${ }^{25}$ already the fact that holes around acceptor defects form small bound polarons challenges the simple explanation of ferromagnetism due to carrier-mediated longrange coupling between magnetic moments residing on cation vacancies. The prototypical example of the cation vacancies in $\mathrm{MgO}$ clearly illustrates this point. Recently, numerous theoretical papers have proposed that $\mathrm{Mg}$ vacancies can induce room temperature ferromagnetism in $\mathrm{MgO} .{ }^{11,13,14}$ Based mainly on DFT LSDA and GGA results, Mg vacancy centers are reported to produce half-metallic shallow acceptor states at the top of the valence band that lead to long range ferromagnetic coupling via a double exchange mechanism.

However, the fact that a number of electron spin resonance (ESR) experiments dating back to the 1970s have clearly established the localized polaronic nature of $\mathrm{Mg}$ vacancies in $\mathrm{MgO}$ (Refs. 26 and 27) seems to have been forgotten. As pointed out by Stoneham et al.,${ }^{21}$ the cation vacancy can be experimentally found in two charging states: neutral, $V^{0}{ }_{\mathrm{Mg}}$, and singly charged, $V_{\mathrm{Mg}}^{-}$. ESR measurements establish that $V_{\mathrm{Mg}} s$, in both the charging states, are deep traps exhibiting states in the gap as opposed to shallow levels at the top of the valence band. In the case of $V_{\mathrm{Mg}}^{0}$, the two holes from the vacancy localize completely on two adjacent oxygen sites. In contrast, in the case of $V_{\mathrm{Mg}}^{-}$, the only hole is completely localized on one single oxygen anion with an accompanying bond distortion. 
TABLE I. Calculated bond lengths (in $\AA$ ), for $V_{\mathrm{Mg}}^{0}$ and $V_{\mathrm{Mg}}$ as calculated by different DFT functionals. Bond lengths correspond to the distance from the center of the $\mathrm{Mg}$ vacancy site to the $\mathrm{O}$ ions in the coordination octahedron. The number in brackets is the number of bonds of a given length.

\begin{tabular}{lcc}
\hline \hline & $V^{0}{ }_{M g}$ & $V_{M g}$ \\
\hline LSDA & $2.19(6)$ & $2.21(6)$ \\
GGA & $2.233(6)$ & $2.24(6)$ \\
ASIC & $2.11(2), 2.22(4)$ & $2.13(1), 2.24(1), 2.25(4)$ \\
HSE & $2.111(2), 2.253(4)$ & $2.106(1), 2.225(1), 2.253(4)$ \\
\hline \hline
\end{tabular}

The availability of ESR experimental data, establishing the nature of $V_{\mathrm{Mg}}$ in $\mathrm{MgO}$, sets a stringent test for DFT predictions obtained with different exchange correlation functionals. In particular, the results of semilocal LSDA/ GGA can be compared to beyond LSDA approaches. In this work, we look at three such methods. The first is the Heyd-Scuseria-Ernzerhof (HSE) screened hybrid density functional, ${ }^{28}$ which represents a promising tool to study many properties of solid state systems, in particular wide-gap oxides. ${ }^{29}$ This was recently shown able to describe correctly the interplay between electronic properties and lattice distortion in oxides as complex as $\mathrm{BaBiO}_{3}{ }^{30}$ The second is the atomic self-interaction correction (ASIC) scheme, which has been employed previously to study a number of oxide and nitride wide-gap materials. ${ }^{31}$ The third is the well known LSDA $+U$ method. ${ }^{32}$

LSDA, ASIC, and LSDA $+U$ calculations were carried out using a development version of the SIESTA code. ${ }^{33}$ Norm-conserving pseudopotentials with the following reference atomic configurations were employed. $\operatorname{Mg}\left([\mathrm{Ne}] 3 \mathrm{~s}^{2}\right)$, $\left([\mathrm{He}] 2 \mathrm{~s}^{2}, 2 \mathrm{p}^{4}\right)$. A double zeta basis set with additional polarization functions was used for both $\mathrm{Mg}$ and $\mathrm{O}$. At the site of the vacancy, extra basis functions corresponding to $\mathrm{Mg}$ atom were introduced to minimize basis set errors. Supercells containing 96 atoms and 192 atoms were used for single defect and multiple defect calculations, respectively. Ionic coordinates were optimized using a conjugate gradients algorithm until all the forces are smaller than $0.04 \mathrm{eV} / \AA$. The GGA and HSE calculations were carried out using the VASP package. ${ }^{34}$ A 54 atom supercell was used to study the $V^{0}{ }_{\mathrm{Mg}}$ center, while a cubic 64 atom supercell was used to simulate the singly charged $V_{\mathrm{Mg}}$ center. A plane wave energy cutoff of $520 \mathrm{eV}$ and a $3 \times 3 \times 3 \quad \Gamma$-centered $k$-point mesh were employed. For the HSE functional, the default value AEXX $=0.25$ is used for the Hartree-Fock exchange mixing parameter and for the screening of the Fock exchange HFSCREEN $=0.2$ is used. Geometry optimization was carried out using conjugate gradients.

We start our analysis by presenting the LSDA results, with the GGA ones being rather similar. The $V_{\mathrm{Mg}}$ site in $\mathrm{MgO}$ presents a perfect octahedral symmetry which is preserved in LSDA, even upon lattice relaxation. This is the case for both $V_{\mathrm{Mg}}^{0}$ and $V_{\mathrm{Mg}}^{-}$. The bond lengths resulting from this isotropic relaxation are presented in Table I. The associated hole density always spreads uniformly over all of the six coordinating $\mathrm{O}$ anions with $p$-orbital lobes pointing toward

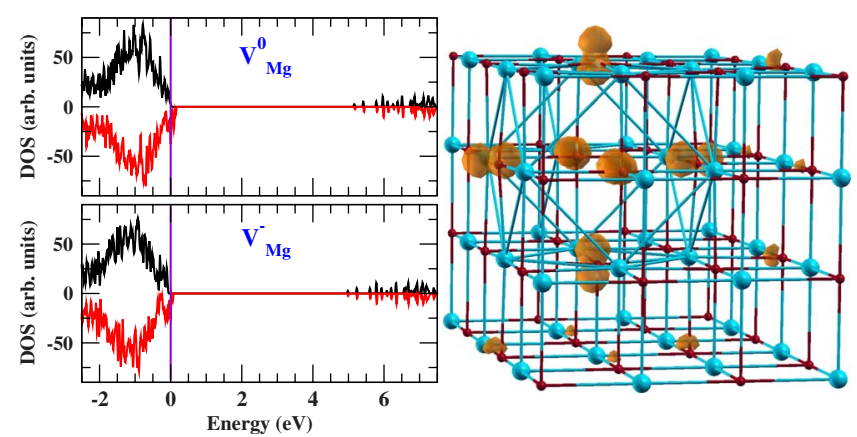

FIG. 1. (Color online) LSDA electronic structure of $V_{\mathrm{MgO}}$. (Left) DOS of a $\mathrm{MgO}$ supercell containing either $V^{0}{ }_{\mathrm{Mg}}$ or $V_{\mathrm{Mg}}^{-}$. (Right) Charge density corresponding to the minority spin holes at the top of the valence band for $V^{0}{ }_{\mathrm{Mg}}$. Note that the hole density is spread uniformly over the $\mathrm{O}$ octahedron.

the center of the vacancy (see Fig. 1). In the case of the $V^{0} \mathrm{Mg}$ a spin triplet, $S=1$, is obtained, while the spin singlet $S=0$ cannot be stabilized. In contrast, $V_{\mathrm{Mg}}^{-}$presents a $S=1 / 2$ ground state. For both the charging configurations the density of states (DOS) is half-metallic as shown in Fig. 1 and corresponds to that of a shallow acceptor impurity with hole states situated at the top of the valence band. This picture however does not conform with the experimental ESR data referenced earlier.

In contrast, a qualitatively different description of the ground state emerges from HSE and ASIC, with the two methods being consistent with each other. First, lattice relaxation around the vacancy site is seen to break the perfect octahedral symmetry. In the case of the $\left(V^{0}{ }_{\mathrm{Mg}}\right)$ center, two virtual bonds from the center of the vacancy to two opposing $\mathrm{O}$ anions are found to shorten with respect to four other bonds forming a plane perpendicular to the two shorter bonds. In the case of the $V_{\mathrm{Mg}}^{-}$site, one of the two previously shorter bonds is seen to elongate while the fourfold symmetry of the bonds in the plane perpendicular to these bonds is retained (see Table I). The symmetry breaking around the vacancy site is also accompanied by a strong localization of the hole density on the $\mathrm{O}$ ions nearest to the vacancy forming small polarons (see Fig. 2). It should be noted that the symmetry lowering around the vacancy site may not occur spontaneously during the course of the geometry optimization, since the octahedrally symmetric solution is a local minimum. Therefore, in order to access the lower symmetry ground state it might be necessary to initialize the geometry optimization in a slightly symmetry distorted ionic configuration around the site.

HSE returns us an energy gain due to the polaronic distortion of $360 \mathrm{meV}$ for $V^{0}{ }_{\mathrm{Mg}}$ and $225 \mathrm{meV}$ for $V_{\mathrm{Mg}}$, and therefore it establishes that the distorted geometry is considerably more stable than the octahedrally symmetric one regardless of the charging state.

The ground state DOS obtained within ASIC and HSE is shown in Figs. 2 and 3. As opposed to the shallow acceptor states observed in LSDA/GGA, both $V^{0}{ }_{\mathrm{Mg}}$ and $V_{\mathrm{Mg}}$ exhibit a deep acceptor level in the gap. This is located at $\sim 1 \mathrm{eV}$ $(\sim 1.25 \mathrm{eV})$ above the valence band maximum $(\mathrm{VBM})$ for HSE (ASIC). It is interesting to note that for the symmetri- 


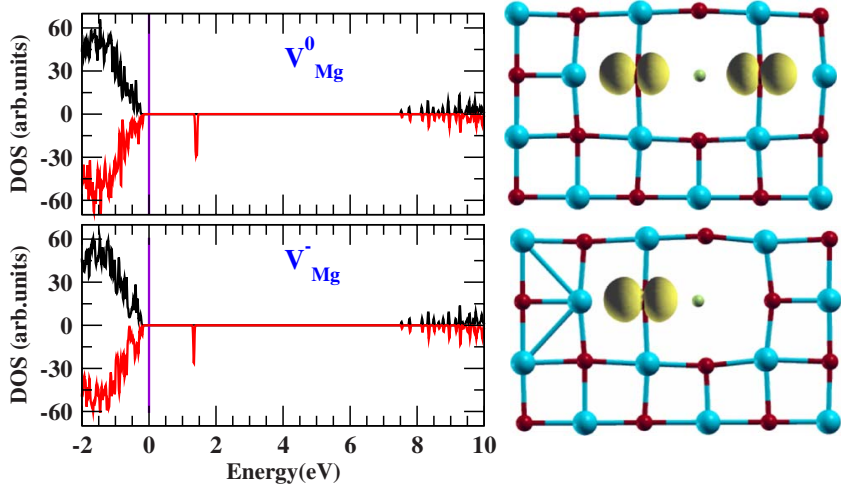

FIG. 2. (Color online) ASIC electronic structure of $V_{\mathrm{MgO}}$ (Left) DOS of a $\mathrm{MgO}$ supercell containing either $V^{0}{ }_{\mathrm{Mg}}$ or $V_{\mathrm{Mg}}$. (Top right) Charge density corresponding to the holes of $V^{0}{ }_{\mathrm{Mg}}$. Note that the two holes are completely localized on two opposing $\mathrm{O}$ ions. (Bottom right) Charge density corresponding to the single hole of $V_{\mathrm{Mg}}^{0}$. The hole density is localized on one $\mathrm{O}$ ion.

cally relaxed local minimum solution within HSE (see lower panels of Fig. 3), a shallow acceptor DOS similar to the LSDA solution is obtained. Thus the deep acceptor ground state is intimately associated to the polaronic bond distortion around the vacancy.

In order to further substantiate the HSE/ASIC results, we also carried out some test calculations within $\mathrm{LSDA}+U$ mainly looking at the evolution of the ground state as a function of the strength of the $U$ parameter. The $U$ correction in this case is applied to the $p$ orbitals of the $\mathrm{O}$ sublattice. For a given value of $U$, the total LSDA $+U$ energies obtained at the LSDA and ASIC geometries presented in Table I are compared. The energy difference $\Delta E=E\left(G_{\mathrm{LSDA}}\right)-E\left(G_{\mathrm{ASIC}}\right)$, where $G_{\mathrm{LSDA}}\left(G_{\mathrm{ASIC}}\right)$ denote the LSDA (ASIC) geometry, is shown in Fig. 4. As the strength of $U$ is increased the bond distorted ASIC geometry is seen to be stabilized with respect to the symmetric LSDA geometry and the crossover is around $U=4 \mathrm{eV}$ for both $V_{\mathrm{Mg}}^{0}$ and $V_{\mathrm{Mg}}$. Also the nature of the defect ground state changes from shallow acceptor to deep acceptor on either side of the crossover (Fig. 4). In

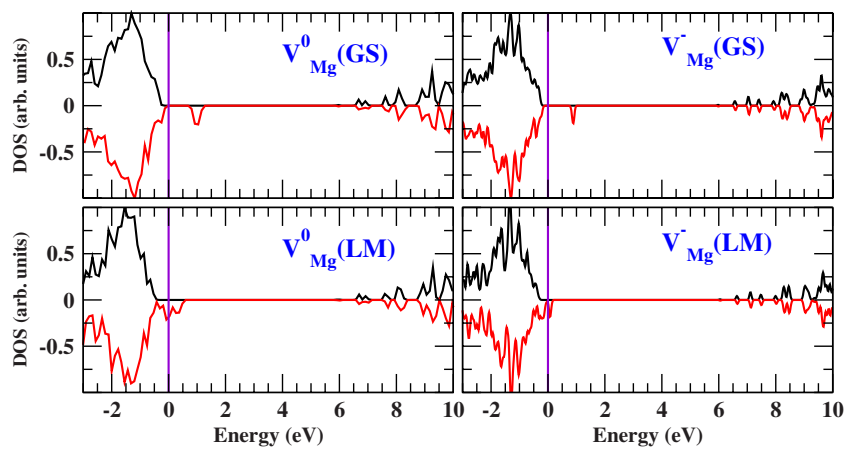

FIG. 3. (Color online) DOS calculated with HSE for a $\mathrm{MgO}$ supercell containing either $V^{0}{ }_{\mathrm{Mg}}$ or $V_{\mathrm{Mg}}$. The top two panels show the DOS for the polaronic ground state geometry (labeled GS) of the defects. The bottom two panels show the DOS corresponding to local minima solutions (labeled LM), which preserve the octahedral symmetry around the vacancy sites.
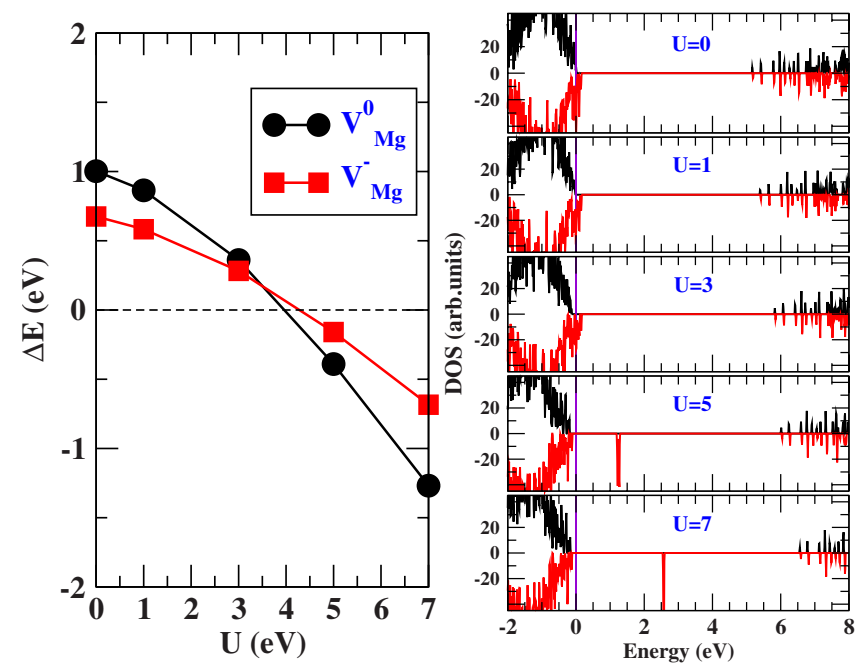

FIG. 4. (Color online) (Left) LSDA $+U$ energy difference between the LSDA and ASIC geometries for $V^{0}{ }_{\mathrm{Mg}}$ and $V_{\mathrm{Mg}}$ as a function of $U$. Note the crossover between the two geometries at $U$ $\sim 4 \mathrm{eV}$, when the polaronic ASIC geometry becomes the most stable. (Right) Ground state DOS calculated with $\mathrm{LSDA}+U$ as a function of $U$.

wide-gap oxides, the value of $U$ for the O- $2 p$ orbitals is estimated to be in the range of $5-7 \mathrm{eV}^{24}$ At $U=5 \mathrm{eV}$, for the $V_{\mathrm{Mg}}^{0}\left(V_{\mathrm{Mg}}\right)$ center the polaronic $G_{\mathrm{ASIC}}$ geometry is lower in energy by a robust $\sim 390 \mathrm{meV}(160 \mathrm{meV})$ with respect to the $G_{\text {LSDA }}$ geometry. Furthermore the DOS for $V^{0}{ }_{\text {Mg }}$ (Fig. 4) exhibits a deep acceptor state located at roughly $1.3 \mathrm{eV}$ above the VBM, which agrees well with the HSE results.

We now look at the magnetic properties associated to $\mathrm{Mg}$ vacancies. For the $V^{0}{ }_{\mathrm{Mg}}$ center, the spins of the two holes may be aligned parallel or antiparallel leading either to a $S$ $=1$ triplet or a $S=0$ singlet. As discussed above, LSDA/GGA predict a stable $S=1$ configuration. However, within HSE/ ASIC, the energies of the $S=1$ and the $S=0$ solutions are found to be very similar, separated only by $0.75 \mathrm{meV}$ (2 meV) in HSE (ASIC). We have also calculated the interdefect magnetic coupling interaction between two $(S=1)$ $V^{0}{ }_{\text {Mg }}$ centers in a 192 atom supercell. For LSDA two sites separated by $8.34 \AA$ are ferromagnetically coupled with a spin-flip energy of $33 \mathrm{meV}$ (the energy required to reverse the spin of one of the two vacancies). This suggests the stabilization of ferromagnetism via magnetic percolation between the holes, provided they are formed at a sufficiently high concentration. In contrast, ASIC predicts for the same supercell configuration, a tiny interaction energy of $1 \mathrm{meV}$. Thus, we find that as soon as the correct polaronic ground state is achieved for the holes, their magnetic interaction virtually disappears.

Before concluding, we add an additional remark on the similarity between $V_{\mathrm{Mg}}$ and Li impurity substituting $\mathrm{Mg}$ $\left(\mathrm{Li}_{\mathrm{Mg}}\right)$. Indeed, both are single acceptors located at the cation sublattice. For $\mathrm{Li}_{\mathrm{Mg}}$ as well, LSDA returns a half-metallic ground state, with the hole completely delocalized on all the $\mathrm{O}$ ions coordinating the impurity. ASIC instead localizes the hole on one $\mathrm{O}$ ion in a distorted polaronic geometry. This is qualitatively identical to what found for $V_{\mathrm{Mg}}^{-}$the $\mathrm{Li}-\mathrm{O}$ bond 
lengths are: $2.31 \AA(1), 1.93 \AA(1), 2.15 \AA(4)]$. Although we did not find an experimental reference to confirm the ground state of $\mathrm{Li}_{\mathrm{Mg}}$ in bulk $\mathrm{MgO}$, its nature as a small bound polaron is well established in the case of surfaces and beyondLSDA electronic structure methods have previously been used to study this defect in the context of catalysis. ${ }^{35}$

In conclusion, we have shown that semilocal LSDA/GGA can lead to qualitative failures in their description of hole centers in $\mathrm{MgO}$. Beyond-LSDA approaches that are either self-interaction free or effectively correct for it, are able to reproduce the experimentally observed polaronic ground state. Significantly, the large intersite ferromagnetic interaction predicted by LSDA is also shown to be an artifact. Thus, the observed high temperature ferromagnetic signals in $\mathrm{MgO}$ cannot be explained by a simple model where magnetic interactions between hole centers, in their ground state, percolate through the sample.

This work was supported by Science Foundation of Ireland and by the EU (ATHENA project). Computational resources have been provided by the Trinity Center for High Performance Computing and by ICHEC.
${ }^{1}$ J. M. D. Coey, Solid State Sci. 7, 660 (2005).

${ }^{2}$ Carbon-Based Magnetism: An Overview of the Magnetism of Metal-Free Carbon-Based Compounds and Materials, edited by T. Makarova and F. Palacio (Elsevier, Amsterdam, 2006).

${ }^{3}$ P. Crespo, R. Litran, T. C. Rojas, M. Multigner, J. M. de la Fuente, J. C. Sanchez-Lopez, M. A. Garcia, A. Hernando, S. Penades, and A. Fernandez, Phys. Rev. Lett. 93, 087204 (2004).

${ }^{4}$ M. Venkatesan, C. B. Fitzgerald, and J. M. D. Coey, Nature (London) 430, 630 (2004).

${ }^{5}$ S. Zhou, E. Cizmar, K. Potzger, M. Krause, G. Talut, M. Helm, J. Fassbender, S. A. Zvyagin, J. Wosnitza, and H. Schmidt, Phys. Rev. B 79, 113201 (2009).

${ }^{6}$ R. P. Panguluri, P. Kharel, C. Sudakar, R. Naik, R. Suryanarayanan, V. M. Naik, A. G. Petukhov, B. Nadgorny, and G. Lawes, Phys. Rev. B 79, 165208 (2009).

${ }^{7}$ Y. Liu, Z. Lockman, A. Aziz, and J. MacManus-Driscoll, J. Phys.: Condens. Matter 20, 165201 (2008).

${ }^{8}$ K. Potzger, S. Zhou, J. Grenzer, M. Helm, and J. Fassbender, Appl. Phys. Lett. 92, 182504 (2008).

${ }^{9}$ C. Chen, L. He, L. Lai, H. Zhang, J. Lu, L. Guo, and Y. Li, J. Phys.: Condens. Matter 21, 145601 (2009).

${ }^{10}$ J. Hu, Z. Zhang, M. Zhao, H. Qin, and M. Jiang, Appl. Phys. Lett. 93, 192503 (2008).

${ }^{11}$ J. I. Beltrán, C. Monty, Ll. Balcells, and C. Martínez-Boubeta, Solid State Commun. 149, 1654 (2009).

${ }^{12}$ N. Kumar, D. Sanyal, and A. Sundaresan, Chem. Phys. Lett. 477, 360 (2009).

${ }^{13}$ F. Gao, J. Hu, C. Yang, Y. Zheng, H. Qin, L. Sun, X. Kong, and M. Jiang, Solid State Commun. 149, 855 (2009).

${ }^{14}$ F. Wang, Z. Pang, L. Lin, S. Fang, Y. Dai, and S. Han, Phys. Rev. B 80, 144424 (2009).

${ }^{15}$ A. Sundaresan, R. Bhargavi, N. Rangarajan, U. Siddesh, and C. N. R. Rao, Phys. Rev. B 74, 161306(R) (2006).

${ }^{16}$ I. S. Elfimov, S. Yunoki, and G. A. Sawatzky, Phys. Rev. Lett. 89, 216403 (2002).
${ }^{17}$ C. Das Pemmaraju and S. Sanvito, Phys. Rev. Lett. 94, 217205 (2005).

${ }^{18}$ A. Droghetti, C. D. Pemmaraju, and S. Sanvito, Phys. Rev. B 78, 140404(R) (2008).

${ }^{19}$ A. Droghetti and S. Sanvito, Appl. Phys. Lett. 94, 252505 (2009).

${ }^{20}$ O. F. Schirmer, J. Phys.: Condens. Matter 18, R667 (2006).

${ }^{21}$ A. M. Stoneham, J. Gavartin, A. L. Shluger, A. V. Kimmel, D. Munõz Ramo, H. M. Rønnow, G. Aeppli, and C. Renner, J. Phys.: Condens. Matter 19, 255208 (2007).

22 J. A. Chan, S. Lany, and A. Zunger, Phys. Rev. Lett. 103, 016404 (2009).

${ }^{23}$ S. Lany and A. Zunger, Phys. Rev. B 80, 085202 (2009).

${ }^{24}$ V. Pardo and W. E. Pickett, Phys. Rev. B 78, 134427 (2008).

${ }^{25}$ J. Osorio-Guillén, S. Lany, S. V. Barabash, and A. Zunger, Phys. Rev. Lett. 96, 107203 (2006).

${ }^{26}$ B. H. Rose and L. E. Halliburton, J. Phys. C 7, 3981 (1974).

${ }^{27}$ L. A. Kappers, F. Dravnieks, and J. E. Wertz, J. Phys. C 7, 1387 (1974).

${ }^{28}$ J. Heyd, G. E. Scuseria, and M. Ernzerhof, J. Chem. Phys. 118, 8207 (2003).

${ }^{29}$ J. Paier, M. Marsman, K. Hummer, G. Kresse, I. C. Gerber, and J. G. Ángyán, J. Chem. Phys. 124, 154709 (2006).

${ }^{30}$ C. Franchini, G. Kresse, and R. Podloucky, Phys. Rev. Lett. 102, 256402 (2009).

${ }^{31}$ C. D. Pemmaraju, T. Archer, D. Sánchez-Portal, and S. Sanvito, Phys. Rev. B 75, 045101 (2007).

${ }^{32}$ V. I. Anisimov, F. Aryasetiawan, and A. I. Lichtenstein, J. Phys.: Condens. Matter 9, 767 (1997).

${ }^{33}$ J. M. Soler, E. Artacho, J. D. Gale, A. García, J. Junquera, P. Ordejón, and D. Sánchez-Portal, J. Phys.: Condens. Matter 14, 2745 (2002).

${ }^{34}$ G. Kresse and J. Hafner, Phys. Rev. B 48, 13115 (1993).

${ }^{35}$ M. Nolan and G. Watson, Surf. Sci. 586, 25 (2005). 